\title{
Modeling of the kinetics of reactive diffusion of gallium in copper particles
}

\author{
S. V. Dzyubanenko ${ }^{1,2}$, V. D. Lukyanov ${ }^{1,2}$ \\ ${ }^{1}$ ITMO University, 49 Kronverkskiy, St. Petersburg, 197101, Russia \\ ${ }^{2}$ JSC Avangard, St. Petersburg, Russia \\ lukyanovvd@rambler.ru
}

PACS 02.30.Hq; 66.30.-h

DOI 10.17586/2220-8054-2015-6-3-332-345

The results of experimental studies for the formation kinetics of an intermetallic compound in the diffusion of gallium in copper are discussed. The mathematical model for the experimental study made it possible to calculate the diffusion coefficient of gallium in copper. A mathematical model for the kinetics of intermetallic compound formation at the reactive diffusion of gallium in a copper particle allows one to estimate the solidification time for diffusion-curing paste-based solder consisting of molten gallium and copper powder.

Keywords: reactive diffusion, solder, gallium, copper, intermetallic, kinetics.

Received: 2 December 2014

Revised: 18 December 2014

\section{Introduction}

Currently, new composite nanostructure materials are being actively introduced in many production areas. The relevance of these materials above all depends on their outstanding mechanical properties. These materials are also actively used in microelectronics.

When assembling electronic equipment, diffusion-curing paste-based solder (DCPS) based on gallium is used; such alloys allow the combining of strength and vacuum density, inherent to solder joints and with the ability of adhesives to cure at ambient temperatures [1-4]. We propose to use the available solder with copper powder filler when preparing diffusioncuring paste-based solder $[5,6]$. The optimum composition of DCPS components, as a result of reactive diffusion of gallium into the particles of copper, provides the formation of an homogeneous copper intermetallic compound $\mathrm{CuGa}_{2}$, which has fast (10 - 60 hours) solidity and possesses high operating temperature (up to $1000^{\circ} \mathrm{C}$ ), which allows the utilization of the product in harsh environments [7-11].

One of the main process parameters characterizing practical properties of paste-based solder is their solidification time, $T_{*}$. The small diameter of the copper powder particle $(5-$ 30 microns) prevents experimental investigation of homogeneous DCPS formation kinetics and in particular, prevents us from estimating the end time for reactive diffusion of interacting components and homogeneous intermetallic formation. In the experiments, another observed factor, $T_{0}$ was under investigation; this factor characterizes an important property of paste-based solder, the formation time of a monolithic DCPS mass that does break into fragments when applying pressure of about $3 \mathrm{~kg} / \mathrm{mm}^{2}$. The load value was selected on the base of the operating conditions for products where DCPS was used. For the studied DCPS composition at the operating temperature $(85 \pm 5)^{\circ} \mathrm{C}$, it was deduced from experiments that $T_{0}=16.5 \pm 0.5$ hour. Let us set a challenge for the mathematical modeling of reactive diffusion of gallium in the copper particles: to evaluate the solidification time of DCPS $T_{*}$ and to match this figure with $T_{0}$ time. 


\section{Experimental study of reactive diffusion of gallium in the copper particles and experimental data analysis}

To justify the choice of mathematical model, an experimental study of gallium and copper reactive diffusion has been carried out. The study of the diffusion layer was carried out on samples of copper metal wire with $3 \mathrm{~mm}$ diameter, incubated for various times ranging from several hours up to seven days at $85 \pm 5{ }^{\circ} \mathrm{C}$ with gallium fusion. After incubation, gallium surplus was removed by means of washing in 2-propanol and distilled water. Wire samples were cut with the help of a slow-precision cutting machine. To form the electron-microscope image of cross-sections, we used signals of secondary and backscattered electrons, allowing us to obtain morphological and compositional contrast, respectively, which depended on the element or phase composition of the sample. Specification of the ultimate sample composition was carried out by means of electron probe microanalysis (EPMA), which is based on comparison of X-ray spectra of the analyzed sample and standards of known composition. The sensitivity of the method is $\sim 0.1 \mathrm{wt} . \%$.

Figure 1 shows a photograph of the section of a cylindrical wire cross-section with intermetallic layer and Fig. 2 shows the results of combined SEM and X-ray microanalysis studies of gallium $\mu$ and copper $1-\mu$ mass content distribution in this section $\mu=M /\left(M+M_{1}\right)$, where $M$ and $M_{1}$ are the mass of gallium and copper in unit volume.

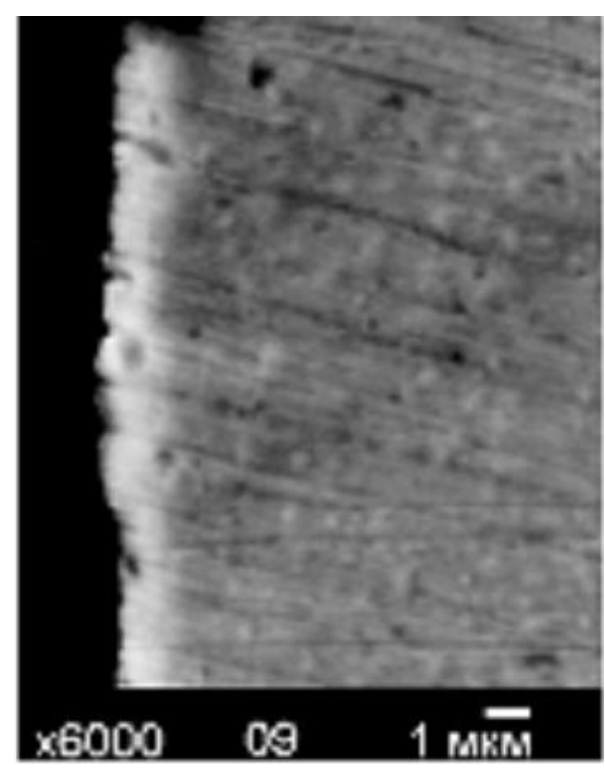

FIG. 1. ross-section of cylindrical wire (light area is a zone of an intermetalli formation)

It becomes clear from the analysis of the experimental data that after bringing gallium and copper in contact in the first short period of time, the duration of which is about $10-$ 20 minutes, the diffusion of gallium into copper without intermetallic formation takes place. At the same time, copper is partially displaced from the gallium particle. Taking into account gallium liquid phase, having its surplus and taking into account the possibility of gallium intermixing, we believe that the concentration of gallium here remains unchanged. After reaching the critical mass of gallium fraction $\mu_{*}=0.63$ on the surface of the particle, intermetallic formation begins to take place [1]. The diffusion of gallium through formed intermetallic compound leads to a moving of the intermetallic compound boundaries deeper into copper. Distribution of gallium, bound in intermetallic compound and freely diffusing through it, depends on the distance from the surface of the copper particles and has a linear relationship at Fig. 2 that is typical 


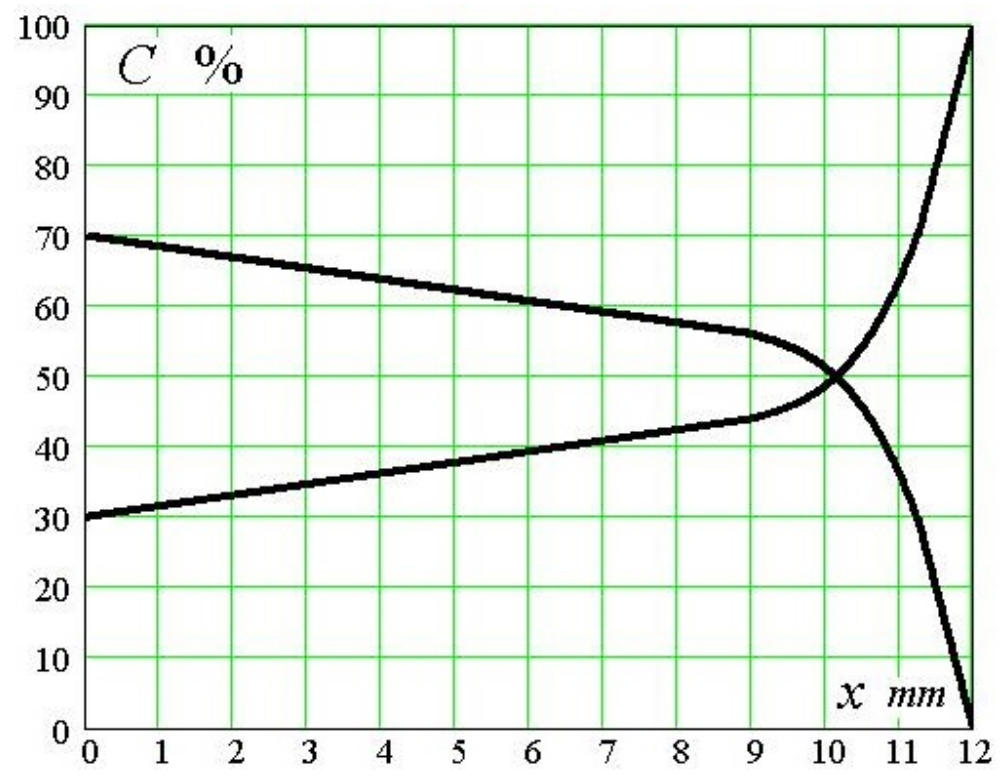

FIG. 2. Experimental data on the distribution of concentration of DCPS components, depending on the distance of gallium penetration into the copper (curve 1 concentration of $\mathrm{Ga}$, curve 2 - concentration of $\mathrm{Cu}$ )

for a stationary diffusion process. This may be because the speed of intermetallic compound boundaries moving deeper into the particle is much less than the speed set by the concentration distribution of gallium in copper.

\section{Determination of diffusion coefficient of gallium in the intermetallic $\mathrm{CuGa}_{2}$}

Copper particles with particle form and dimensions of $3-4 \mathrm{~mm}$ were used in the experiment. All diffusion processes occur only near the surface of the particle; penetration depth was $150-200 \mu \mathrm{m}$. Therefore, by means of mathematical modeling, we are solving initialboundary one-dimensional, by Cartesian coordinate, non-stationary problem for the diffusion equation of gallium into the half-space filled with copper.

\section{Mathematical modeling of one-dimensional reactive diffusion of liquid gallium in copper}

Suppose at a moment of time, $t=0$, the half-space $x<0$ is filled with liquid gallium and the half-space $x>0$ is filled with solid copper. For a while, there is a diffusion process of gallium in copper, which leads to the appearance of free gallium in the half-space $x>0$. The experiments showed that the duration of this process is small, less than an hour. From a certain moment, an intermetallic $\mathrm{CuGa}_{2}$ component is starting to be formed in copper from the surface and goes deeper. This time $t$ is supposed to be the initial $(t=0)$ and the count shall be carried out starting from it.

The distribution of gallium in copper shall be described by the task at the point $x$ at a point in time, $t$, concentration value of gallium in copper $C=C(x, t)$, which is a dimensionless value that is equal to the mass of gallium $M$ in the volume $V_{0}$, normalized to the bulk density of gallium $\rho: C=M / \rho V_{0}$, if we consider that $M=\rho V$, where $V$ is the value of the volume occupied by gallium, the concentration of gallium is the volume fraction of gallium in unit volume $V_{0}, C=V / V_{0}$. 
When measuring the concentration $C$, we consider the gallium that comprises the intermetallic compound - its concentration is $C_{*}$, and the gallium that continues to diffuse through the intermetallic $[1,3]$. The critical concentration of gallium $C_{*}$ in formation of intermetallic compound is evaluated through a critical mass fraction $\mu_{*}: C_{*}=\frac{\mu_{*} \rho_{1}}{\left(1-\mu_{*}\right) \rho+\mu_{*} \rho_{1}}$, where $\rho_{1}$ is the density of copper. If we consider that $\rho=5910 \mathrm{~kg} / \mathrm{m}^{3}, \rho_{1}=8920 \pm 10 \mathrm{~kg} / \mathrm{m}^{3}$, $\mu_{*}=0.63 \pm 0.02$, then $C_{*}=0.76 \pm 0.02$.

The thickness of intermetallic compound grows with the time; its leading edge has $x=\xi$ coordinate.

The diffusion process of gallium into the intermetallic compound with $0<x<\xi$ is described by the second Fick's law $[1,12,13]$ :

$$
\frac{\partial C}{\partial t}=D \frac{\partial^{2} C}{\partial x^{2}}
$$

where $D$ is the diffusion coefficient of gallium into the intermetallic compound. conditions:

Equation (1) shall be solved with the initial condition $C_{1}(x, 0)=0$ and boundary

with $x=0$ :

$$
-D \frac{\partial C}{\partial x}(0, t)=\alpha\left(C_{0}-C(0, t)\right)
$$

where $\alpha$ is the coefficient of diffusion permeability of gallium into the intermetallic compound, $C_{0}=1$ the concentration of gallium in the half-space $x<0$;

with $x=\xi$ :

$$
C_{1}(\xi, t)=C_{*} .
$$

This boundary condition defines the condition for the formation of the intermetallic compound at its front.

If we consider the case $\alpha \rightarrow+\infty$ - full penetration of gallium into intermetallic, then condition (2) leads to the boundary condition:

$$
C(0, t)=C_{0} .
$$
equation:

The movement of the intermetallic front $\xi=\xi(t)$ with $t>0$ is given by differential

$$
C_{*} \frac{d \xi}{d t}=-D \frac{\partial C_{1}(\xi-0, t)}{\partial x},
$$

which is obtained by equating the amount of gallium $d m_{1}(t)=C_{*} d \xi$ at the unit area of intermetallic front of gallium and the quantity of gallium flowing through a unit area per unit time

$$
d m_{2}(t)=-D \frac{\partial C_{1}(\xi-0, t)}{\partial x} d t
$$

The differential equation (5) we solve with the initial condition

$$
\xi(0)=0 .
$$

Analysis of the experimental curves (Fig. 2) shows that the distribution of concentration of gallium in the intermetallic layer is close to stationary when in equation (1) the value $\partial C / \partial t$ can be neglected and the equation becomes $d^{2} C / d x^{2}=0$. The general solution of this equation is a linear function along the $x$ coordinate. 
The approximate solution of equation (1) by Leibenson $[14,15]$ we are looking for in a 'quasi-static' form when there is a linear dependence on $x$ coordinate with coefficients that depend on time as a parameter:

$$
C(x, t)=A(t) x+B(t)\left(1-\frac{x}{\xi(t)}\right) .
$$

Using boundary conditions (4) and (5), we shall express functions $A(t)$ and $B(t)$ through the unknown function :

$$
A(t)=\frac{C_{*}}{\xi(t)}, \quad B(t)=\frac{\alpha \xi(t) C_{0}+D C_{*}}{\alpha \xi(t)+D} .
$$

For the $\xi(t)$ function, we shall obtain the differential equation of the first order, if we substitute the representation (7), taking into account formula (8):

$$
C_{*} \frac{d \xi}{d t}=\alpha D \frac{C_{0}-C_{*}}{\alpha \xi+D}
$$
form:

A particular solution of differential equation (9), satisfying initial condition (6) has the

$$
t=\frac{C_{*}}{C_{0}-C_{*}}\left(\frac{1}{2 D} \xi^{2}+\frac{1}{\alpha} \xi\right)
$$
solution:

An ultimate situation of complete penetration, when $\alpha \rightarrow+\infty$ we get a particular

$$
t=\frac{C_{*}}{2 D\left(C_{0}-C_{*}\right)} \xi^{2}
$$

or after the solution relative to the intermetallic front coordinate, we shall get the famous [2] law of displacement of the front of the phase transition:

$$
\xi=\sqrt{2 D\left(\frac{C_{0}}{C_{*}}-1\right) t} .
$$

Comparing the theoretical dependences (11) and (12) shows that counting of final permeability at the boundary of liquid gallium and copper leads to a change in the coordinate position of the intermetallic, depending on the time in comparison with the case of full penetrability. The main difference we have in the initial stage of the diffusion process, for example, the speed of front moving in the initial time at full permeability is infinite, in case of finite permeability, this speed is finite.

\section{Calculation of diffusion coefficient of gallium in the intermetallic}

Consideration of mutual leakage at the border of liquid gallium and copper allows one, from the range of particular solutions (11) and (12), to hypothesize that the experimental data for the dependence of penetration depth $\xi=\xi(t)$, shown in Fig. 3 with the help of dots have to be approximated by a quadratic function of the form in the case of finite permeability [16],

$$
t=a \xi^{2}+b \xi
$$

or in the case of complete penetration:

$$
t=a \xi^{2} .
$$




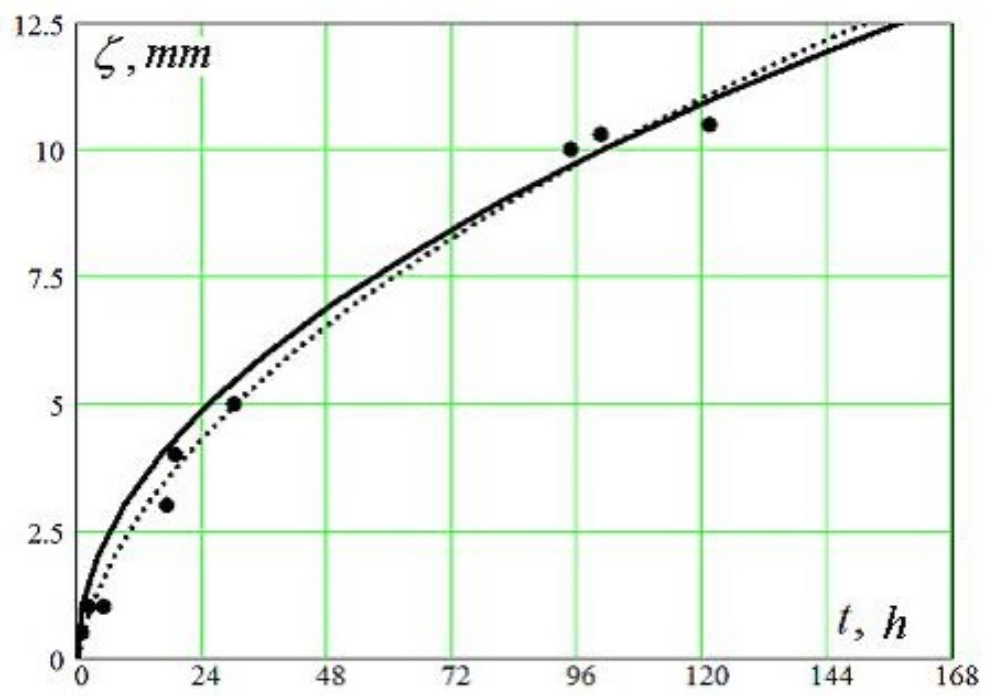

FIG. 3. Dependence of the intermetallic layer depth on the time of sample exposure in gallium. The dots show the experimental date, the dotted line is the dependence diagram (10), continuous line - the dependence diagram (11).

We shall find coefficients in these solutions, using the least-squares method.

Calculations on the data from Fig. 3 give the following values for the coefficients:

- for dependence of type (13) - $a=48.09, b=123.8$;

- for dependence of type (14) $-a=61 \pm 1$.

Comparison of the dependence diagrams in Fig. 3 and numerical values of diffusion coefficients in the case of finite permeability $(\alpha<+\infty)$ and full permeability $(\alpha \rightarrow+\infty)$ shows that account of final permeability is essential for the initial stage of the process; with the large time and deep penetration of intermetallic it can be limited to the case of full penetration.

Comparing formulas (13) and (16) leads to the equation:

$$
\frac{C_{*}}{2 D\left(C_{0}-C_{*}\right)}=a,
$$

to calculate the diffusion coefficient of gallium in intermetallic:

$$
D=\frac{C_{*}}{2 a\left(C_{0}-C_{*}\right)} \text {. }
$$

Value $C_{*}=0.76 \pm 0.02[4]$, we have $D=1.38 \pm 0.12 \mathrm{~mm}^{2} / \mathrm{h}$.

\section{Reactive diffusion of molten gallium in copper particle}

Let assume that copper particle of radius $R$ is put into molten gallium. Reactive diffusion of gallium into the copper particle begins. Assuming spherical symmetry of the process, we have the formation of a spherical layer of the intermetallic, starting from the surface of the copper particle and going deeper into the particle.

Let the radius of the copper particle, in which gallium has not penetrated, be $\zeta$. We assume that the steady-state diffusion of gallium into the copper particle is described by the equation of diffusion for gallium concentration in copper $C=C(r, t)$ at a moment of time $t$ at the point with $r$ coordinate of spherical coordinate system, the benchmark of which is located in the center of the copper particle and that in the absence of concentration of spherical angles has the following form: 


$$
\frac{d^{2} C}{d r^{2}}+\frac{2}{r} \frac{d C}{d r}=0
$$

with $\zeta<r<R$.

The boundary condition on the surface of the copper particle with $r=R$ setting a condition of full permeability of gallium into copper:

$$
C(R, t)=C_{0}(t)
$$

where $C_{0}(t)$ function determines the law of concentration variation of gallium outside a copper particle.

At the intermetallic front with $r=\zeta$, gallium concentrations equals $C_{*}$, ensuring the condition of intermetallic formation:

$$
C(\zeta, t)=C_{*} .
$$

The equation of intermetallic front motion determines the law of front coordinates variation in time $\varsigma=\varsigma(t)$ and has with $r=\zeta$ and $t>0$ the following form:

$$
C_{*} \frac{d \zeta}{d t}=-D \frac{\partial C}{\partial r}(\zeta, t)
$$

with initial condition:

$$
\zeta(0)=R
$$

The general solution of differential equation (15),

$$
C(r)=B-\frac{A}{r} \text {. }
$$

The approximate solution of diffusion equation, according to solutions (20) after the method of Leibenson $[14,15]$ will be found in the 'quasi-static' form:

$$
C(r, t)=B(t)-\frac{A(t)}{r}
$$

Satisfying the boundary conditions (16) and (17), we obtain a system of algebraic linear equations in order to express the functions $A(t)$ and $B(t)$ through coordinates of mobile front of the intermetallic compound $\zeta=\zeta(t)$ :

$$
\left\{\begin{array}{c}
B(t)-\frac{A(t)}{R}=C_{0}(t), \\
B(t)-\frac{A(t)}{\zeta(t)}=C_{*} .
\end{array}\right.
$$

From this system of equations we obtain:

$$
A(t)=\frac{R\left(C_{0}(t)-C_{*}\right) \zeta(t)}{R-\zeta(t)}, \quad B(t)=\frac{R C_{0}(t)-C_{*} \zeta(t)}{R-\zeta(t)} .
$$

Substitution of formula (21) for the distribution of the gallium concentration in the copper particle, taking into account the obtained formulas (23) into the equation of intermetallic front motion (18) leads to a differential equation of intermetallic boundary motion at the counter reactive diffusion of liquid gallium in the copper particle: 


$$
\frac{d \zeta}{d t}=-\frac{D}{C_{*}} \frac{\left(C_{0}(t)-C_{*}\right)}{(R-\zeta(t)) \zeta(t)}
$$

To solve the differential equation (24) with separable variables it is necessary to set the law of concentration variation $C_{0}(t)$.

\section{The time of completion of counter reactive diffusion in the copper particle with an unlimited quantity of molten gallium}

Let us assume that the copper particle is surrounded by such an amount of gallium that its diffusion into copper and repression of copper from the particle does not change the concentration of gallium $\left(C_{0}(t)=1\right)$ in the ambient space of the particle. The differential equation (24) in this case has the form:

$$
C_{*} \frac{d \zeta}{d t}=-D \frac{\left(1-C_{*}\right) R}{\zeta(t)(R-\zeta(t))} .
$$

Analysis of the right side of the differential equation shows that with $\zeta \rightarrow R-0$ and $\zeta \rightarrow 0+0$ the intermetallic front moving speed is striving to $+\infty$ that indicates inapplicability of the obtained equation in these cases. To simulate the diffusion process in these two cases, instead of (16) one must use the boundary conditions with the final permeability of the form (3). Integration of the equation (25) with initial condition (19) gives the particular integral:

$$
t=\frac{C_{*} R^{2}}{\left(1-C_{*}\right) D}\left(\frac{1}{6}-\frac{\zeta^{2}}{2 R^{2}}+\frac{\zeta^{3}}{3 R^{3}}\right) .
$$

The diffusion process is over, if the front reaches the center of the particle and the copper particle becomes intermetallic. The time of diffusion completion, $t_{*}$, shall be calculated if we add the value $\zeta=0$ into the solution (26) and we get:

$$
t_{*}=\frac{C_{*} R^{2}}{6\left(1-C_{*}\right) D} .
$$

This time increases quadratically with increase of the radius of the copper particle and is inversely proportional, depending on the diffusion coefficient, $D$.

\section{Ratio of the components required to produce intermetallic $\mathrm{Ga}_{2} \mathrm{Cu}$}

We are carrying out mixing of molten gallium with $M$ mass and copper powder with $M_{1}$ mass in a vessel. The condition for full use of the components in order to form an intermetallic has the form:

$$
\mu_{*}=\frac{M}{M+M_{1}} .
$$

We assume that the copper powder consists of $N$ identical spherical particles with radius $R$, the amount of which is which we uniformly distribute in gallium.

Using formula $M_{1}=\rho_{1} V_{1} N$, where $\rho_{1}$ is the copper density, we shall calculate the number of copper particles $N=M_{1} / \rho_{1} V_{1}$. For every copper particle, there is such an amount of gallium, which occupies a volume of $V=M / \rho N$.

Let's divide the mixture of substances into $\mathrm{N}$ cubes, the side length of which equals $2 \mathrm{H}$ and the volume is $V_{0}=8 H^{3}$. Each cube falls at exactly one particle of copper. We have the correlation between the volumes $V_{0}=V+V_{1}$. 
Let's place all the gallium, which occupies the volume $V$ nearby this particle in a certain equivalent spherical layer, having the same volume. The radius of the spherical layer varies from $R$ to $R_{0}$, where $R$ is the radius of the particle; $R_{0}$ is the outer radius of spherical layer.

From the conditions of intermetallic formation, with this amount of gallium and copper, we shall find the ratio between $R$ and $R_{0}$.

For the volume of spherical layer filled with gallium, we have $V=4 \pi\left(R_{0}^{3}-R^{3}\right) / 3$. We shall use formula (28), which can be written as:

$$
\mu_{*}=\frac{\rho V}{\rho V+\rho_{1} V_{1}} .
$$

After elementary transformations for the ratio of the radii, we shall obtain

$$
\frac{R_{0}}{R}=\sqrt[3]{1+\frac{\rho_{2}}{\rho_{1}} \cdot \frac{\mu}{1-\mu}},
$$

or the same radii ratio expressed in terms of the critical concentration of gallium $C_{*}$

$$
\frac{R_{0}}{R}=\frac{1}{\sqrt[3]{1-C_{*}}} .
$$

Such ratio of radii of the outer spherical layer and of the copper particle filled with gallium, when a complete intermetallic formation without any surplus takes place, one may call 'intermetallic' $\mathrm{Ga}_{2} \mathrm{Cu}$.

\section{Counter reactive diffusion with limited number of gallium}

We observe the copper particle of radius $R$ covered with spherical layer of gallium, external radius of this layer equal to $R_{0}$.

When the diffusion of the surrounding gallium in copper particle at a moment of time $t$ has a concentration distribution of the form (26) established, functions $A(t)$ and $B(t)$ comprising this formula are given by formula (28).

Using the law of perdurability, we shall find a relation between two unknown quantities, $\zeta(t)$ and $C_{0}(t)$.

To do this, we shall calculate the amount of gallium $\Delta M=\Delta M(t)$ that left the spherical layer and diffused into the copper particle:

$$
\Delta M=\frac{4}{3} \pi\left(R_{0}^{3}-R^{3}\right)\left(1-C_{0}(t)\right) \rho .
$$

We shall calculate the same amount of gallium that comes into the copper particle, having the formula for gallium concentration distribution (21). To do this, we integrate the distribution of gallium concentration on the spherical layer $T$, occupied by intermetallic:

$\Delta M(t)=\rho \iiint_{T} C d V=4 \pi \rho \int_{\zeta(t)}^{R} C_{1}(t, r) r^{2} d r=4 \pi \rho\left(B(t) \frac{\left(R^{3}-\zeta^{3}(t)\right)}{3}-A(t) \frac{\left(R^{2}-\zeta^{2}(t)\right)}{2}\right)$.

Equating the right sides of formulas (30) and (31), taking into account formula (23), we obtain a linear equation for the unknown quantity $C_{0}(t)$ : 


$$
\begin{gathered}
\frac{4}{3} \pi\left(R_{0}^{3}-R^{3}\right)\left(1-C_{0}(t)\right)= \\
=4 \pi\left(\frac{C_{0}(t) R-C_{*} \zeta(t)}{R-\zeta(t)} \cdot \frac{\left(R^{3}-\zeta^{3}(t)\right)}{3}-\frac{\left(C_{0}(t)-C_{*}\right) \zeta(t)}{R-\zeta(t)} \cdot \frac{\left(R^{2}-\zeta^{2}(t)\right)}{2}\right) .
\end{gathered}
$$

The solution of the equation, relative to the function $C_{0}(t)$, leads to correlation, containing the desired function

$$
C_{0}(t)=C_{*}+\frac{R_{0}^{3}\left(1-C_{*}\right)-R^{3}+C_{*} \zeta^{3}(t)}{R_{0}^{3}-0.5 R \zeta(t)(R+\zeta(t))}
$$

\section{Completion time of counter reactive diffusion in copper particle with 'intermetallic' ratio of gallium and copper}

Let us consider the process of DCPS formation that is completed by full conversion of copper and gallium into intermetallic. Here, we have the formula (29), from which we have the equality $R_{0}^{3}\left(1-C_{*}\right)-R^{3}=0$ and formula (32) shall be transformed as:

$$
C_{0}(t)=C_{*}+\frac{C_{*} \zeta^{3}(t)}{R_{0}^{3}-0.5 R \zeta(t)(R+\zeta(t))} .
$$

The equation of front motion of intermetallic formation (24) in view of the above formula (33) for $C_{0}(t)$ function has the following form:

$$
\frac{d \zeta}{d t}=-\frac{D \zeta^{2}(t)}{(R-\zeta(t))\left(R_{0}^{3}-0.5 R \zeta(t)(R+\zeta(t))\right)} .
$$

For the particular solutions of these differential equations with multiple variables with the initial condition (19) we have:

$$
t=t(\zeta)=\frac{R^{2}}{D}\left(\frac{R^{2}-\zeta^{2}}{4 R^{2}}+\left(\frac{1}{2}+\frac{R_{0}^{3}}{R^{3}}\right) \ln \frac{\zeta}{R}+\frac{R_{0}^{3}}{R^{3}} \cdot \frac{R-\zeta}{\zeta}\right)
$$

Note that the function $t=t(\zeta)$ with $0<\zeta \leq R$ is monotonic and has backward $0<\zeta \leq R$.

\section{Analysis and comparison of the results for mathematical modeling of counter reactive diffusion kinetics of gallium in copper}

The time $t$ dependence diagram on the penetration depth into the copper particle of intermetallic front $h=h(t)=1-\zeta(t)$ is shown on Fig. 4 by a solid line. Subsequent calculations were carried out for a particle of $5 \mu \mathrm{m}$ radius, with a value of $C_{*}=0.76$ when, according to formula (29) for the radius of the equivalent particle we have $R_{0}=1.609 R$, $D=1.38 \pm 0.12 \mu \mathrm{m}^{2} / \mathrm{h}$.

For comparison, Fig. 4 shows a diagram of dependence on the depth of penetration into the copper particle at an unlimited quantity of gallium around it (dashed line). When constructing this graph, the function inverse to the function of $\zeta=\zeta(t)$ was used, that defined the formula (26).

The dotted line at Fig. 4 shows the dependence of the depth of penetration into the copper intermetallic front $h=\xi(t)$ in the 2D problem, here, the function $\xi(t)$ is given by the formula (12).

Figure 5 is a diagram of dependence $C_{0}=C_{0}(\zeta(t))$; when specifying this dependence, we use formula (33) and find the dependence $\zeta=\zeta(t)$. This dependence shows the change 


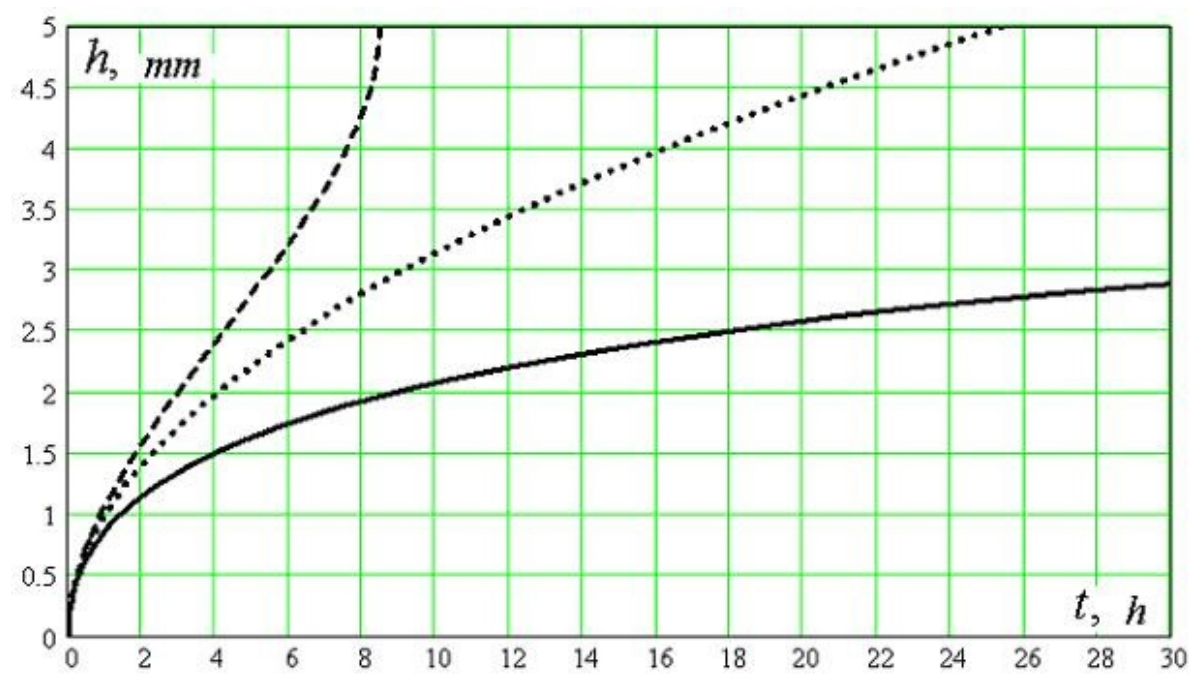

FIG. 4. The time $t$ dependence of the relative depth of intermetallic front penetration into the copper particle. The solid line is the 'intermetallic' structure of gallium around the copper particle; the dotted line is an unlimited quantity of gallium around the copper particle, the dashed line - for a plane copper-gallium boundary

of gallium concentration in time in the spherical layer around the copper particle. When constructing this graph, parametric function $C_{0}=C_{0}(\zeta(t))$ solution is used, $\zeta$ acts as a parameter value, and the functions are defined by formulas (33) and (34):

$$
\left\{\begin{aligned}
t & =t(\zeta), \\
C_{0} & =C_{0}(\zeta) .
\end{aligned}\right.
$$

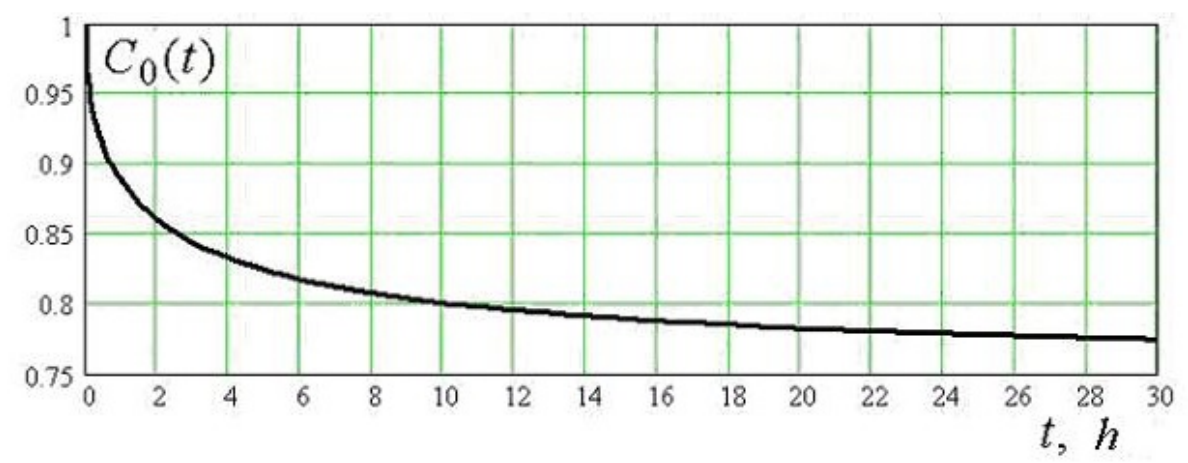

FIG. 5. The time $t$ dependence of the normalized concentration of gallium in the spherical layer $C_{0}(t)$

For distribution of the concentration of gallium in copper particle at the moment of time $t=t_{0}$ we have:

$$
C\left(r, t_{0}\right)=\frac{C_{0}\left(\zeta_{0}\right)-C_{*} \zeta_{0}}{R-\zeta_{0}}-\frac{\left(C_{0}\left(\zeta_{0}\right)-C_{*}\right) \zeta_{0}}{\left(R-\zeta_{0}\right) r},
$$

with $\zeta_{0}<r<R, \zeta_{0}=\zeta\left(t_{0}\right)$. The graph of function $C=C\left(r, t_{0}\right)$ at some points of time are shown in Fig. 6. 


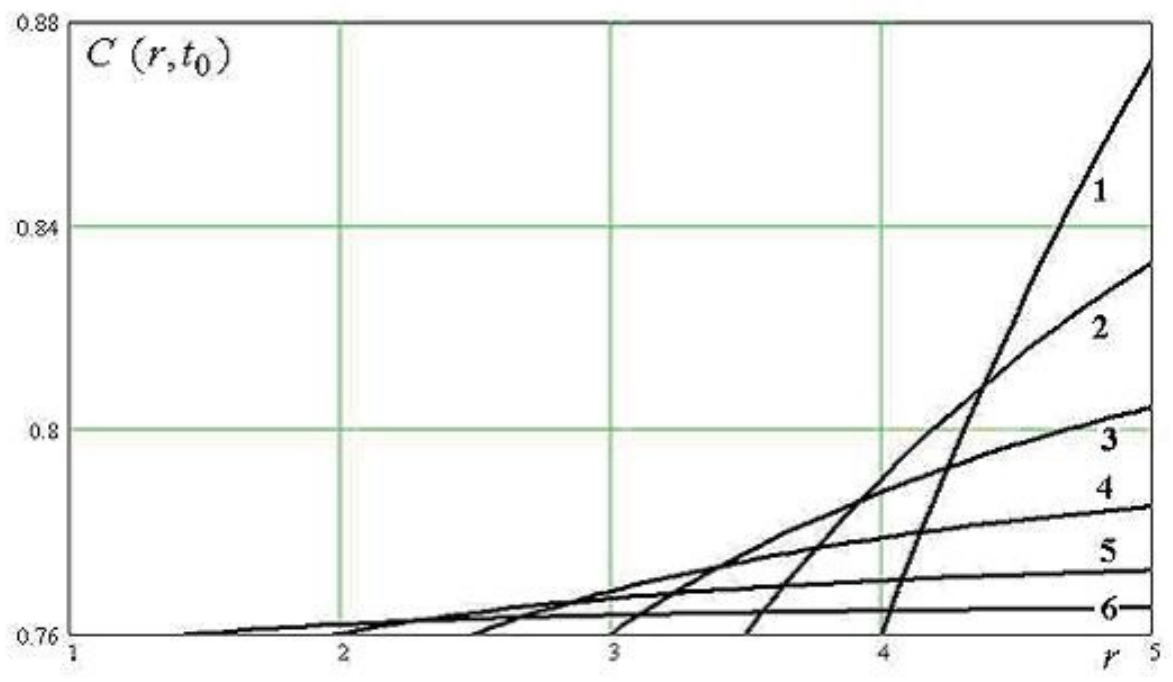

FIG. 6. Distribution of the normalized gallium concentration $C\left(r, t_{0}\right)$ in the copper particle at different values of dimensionless time $t_{0}$. (Curve 1: $t_{0}=1.5$ hours, curve 2: $t_{0}=4.0$ hours, and the curve $3: t_{0}=9.0$ hours, curve 4 : $t_{0}=18.0$ hours; curve $5: t_{0}=35.0$ hours, curve $6: t_{0}=70.0$ hours)

\section{Evaluation of DCPS formation time}

Analysis of the dependences shown in Fig. 4 allows us to estimate the time of intermetallic formation in two cases: in the $2 \mathrm{D}$ problem and in case with an unlimited amount of gallium. If we consider that the radius of the copper particle was chosen to be 5 microns, the time of process completion in case of an unlimited quantity of gallium round the copper particle equals to $T_{1}=8.5$ hours. This time is considerably smaller than the time $=26$ hours the time of entry into the intermetallic front at the $2 \mathrm{D}$ problem to the depth of $5 \mu \mathrm{m}$. This is due to the fact that during diffusion process, while penetrating deeper into the particle, gallium flux density is increased by reducing the area of the sphere, separating the intermetallic from the copper particle. In the $2 \mathrm{D}$ problem, the section that is separating the intermetallic from the copper has a permanent area.

In the case of 'intermetallic' composition, the time of complete conversion of the components into intermetallic compound equals to infinity. Let's determine the value $T$ - the time of practical completion of reactive diffusion or the DCPS formation time to be the time, at which the volume fraction of the intermetallic compound formed throughout the volume of the paste-based solder, equals to some critical value.

By the time $T$, the depth of penetration of intermetallic front $h(T)$ and the amount of formed intermetallic:

$$
v=v(T)=V\left(1-(1-\alpha(T))^{3}\right),
$$

where $\alpha(T)$ - the proportion of penetration depth at $R$ radius, $\alpha=\alpha(T)=h(T) / R$. Concentration of gallium in the spherical layer around the copper particle reaches $C_{0}(T)>C_{*}$. Concentration of copper in this layer equals $1-C_{0}(T)$. This concentration of copper is not enough for intermetallic to be formed in the whole layer, as it is required by $1-C_{*}$ concentration. We shall calculate volume, where this amount of copper will be enough for intermetallic formation: 


$$
v_{1}=\frac{1-C_{0}(T)}{1-C_{*}}\left(V_{0}-V\right) .
$$

Let us calculate the volume fraction of the intermetallic compound $v+v_{1}$ in $V_{0}$ volume that is the volume of the particle, containing all the gallium and copper particles: $\left(v+v_{1}\right) / V_{0}$. The required time $T$ is determined from the equation $\left(v+v_{1}\right) / V_{0}=\gamma$, using the formulas (29), (35) and (36) it takes the form:

$$
\left(1-(1-\alpha)^{3}\right)\left(1-C_{*}\right)+\left(1-C_{0}(T)\right) \frac{C_{*}}{1-C_{*}}=\gamma .
$$

The value we shall find using the given experimental result: DCPS formation time is 16 hours. From Fig. 4 and 5 we shall find the values of $\alpha(16) \approx 0.50 \pm 0.03$ and $C_{0}(16)=$ $0.78 \pm 0.02$. Taking into account these values and having the value $C_{*}=0.76$, we shall calculate the value $\gamma \approx 0.90 \pm 0.02$

Thus, the time for which $90 \%$ of paste turns into intermetallic is understood to be the time of DCPS formation.

\section{Conclusion}

1. An experimental study of the kinetics of reactive diffusion of gallium and copper has been carried out. The experimental and analytical method for determination of the diffusion coefficient of gallium into intermetallic has been proposed; this method allowed us to determine the gallium diffusion coefficient into the intermetallic after building a mathematical model. It was shown that account of final permeability of gallium into copper insignificantly affects the kinetics of the diffusion process, except for a short initial period of time.

2. A theoretical study of the reactive diffusion kinetics of gallium in the copper particle was also done. The differential equation of intermetallic front moving in the copper particle was obtained, on condition of spherical symmetry and arbitrary law of gallium concentration change in the ambient space of the particle.

3. Analytical solutions for the differential equations of intermetallic front moving in the copper particle at a constant concentration of gallium in the space surrounding the particle have been found.

4. An analytical solution for the differential equation of intermetallic front moving in the copper particle, when a limited amount of 'intermetallic' gallium is near. This solution simulates the kinetics of formation of diffusion-curing paste-based solder.

5. A study of constructed analytical solutions has been carried out, which allowed understanding of the reactive diffusion kinetics of gallium and copper microparticles. Taking into account the results of the pilot study, the concept of diffusion-curing paste-based solder formation has been introduced.

\section{References}

[1] Lashko N.F., Lashko S.V. Soldering of metals. Mechanical engineering, Moscow, 1988, 326 p.

[2] Petrova A.P. Heat-resistant glues. Chemistry, Moscow, 1977, 200 p.

[3] Lashko S.V., Grishin V.L. Solders with gallium. Instrumentation making, 1965, 3, P. 6-11.

[4] Grzhimalsky L.L. Gallic solders. Leningrad: Knowledge, 1974.

[5] MacKay C.A. Amalgams as alternative bonding materials. Soldering \& Surface Mount Technology, 1990, 2, P. 21-25.

[6] Baldwin D.F. Amalgams As Alternative Microelectronic Interconnect Materials. Sensors in Electronic Packaging, 1995, 2, P. 55-63. 
[7] Bhattacharya S.K. A low temperature processable ternary gallium alloy for via filling application in microelectronic packaging. Journal of Materials Science: Materials in Electronics, 2000, 11, P. 653-656.

[8] Janssen Y. Differential thermal analysis and solution growth of intermetallic compounds. Journal of Crystal Growth, 2005, 285, P. 670-680.

[9] Deng Yue-Guang. Corrosion development between liquid gallium and four typical metal substrates used in chip cooling device. Appl. Phys. A, 2009, 95 (3), P. 907-915.

[10] Sommadossi S. Diffusion soldering using a gallium metallic paste as solder alloy: study of the phase formation systematic. Journal of Materials Science, 2007, 42, P. 9707-9712.

[11] Dzyubanenko S. Gallium Amalgam Solder as a Bonding Material for SAW Devices. Proceeding of "XIII International Conference for Young Researches 'Wave Electronics and Its Applications in the Information and Telecommunication Systems”. Saint-Petersburg, June 2010, Saint-Petersburg State University of Aerospace Instrumentation, 2010, P. 34.

[12] Bugakov V.Z. Diffusion in metals and alloys. Moskow, GITTL, 1949, 122 p.

[13] Tikhonov A.N., Samarsky A.A. Equations of mathematical physics. Science, Moskow, 1977, 734 p.

[14] Leybenzon L.S. Handbook of oil-field mechanics. Moskow - Leningrad: ONTI NKTP USSR, 1934,196 p.

[15] Luikov A.V. Heat conductivity theory. Moskow, The Higher school, 1966, 600 p.

[16] Lukyanov V.D. Relationship plurality approximation. Nanosystems: Physics, Chemistry, Mathematics, 2014, 5 (3), P. 384-390. 